\title{
Use Google Sketch up As an Illustration in Industrial Drawing
}

\author{
Assad Khudair Daraj ${ }^{\mathrm{a}}$ \\ Ahmed Hameed Shakir ${ }^{b}$ \\ ${ }^{a}$ Al Haritha Industrial High School/ Vocational Education Section / Basra \\ ${ }^{b}$ University of Al-Muthna \\ asaadalmyahi@yahoo.com \\ ahhash13@google.com
}

Keywords: Sketch Up program, Geometric drawing, 3D drawing.

\begin{abstract}
When it comes to drawing 3D shapes, we do not get any easier than the Google Sketch Up program. The most important part of 3D drawing, especially in the field of architecture, is the ease of using the program, the ability to turn the idea into virtual reality, the Google sketch program Up, provides a user-friendly service that is not required for a powerful computer, and we all know that AutoCAD is one of the most powerful 3D drawing programs (if not the most powerful) but the difficulty of using it and the time it takes to learn has led most engineers to resort to the easiest program. indispensable but Google sketch programs gave up solutions faster and many choices in final directing. The study examined the problem of the use of AutoCAD program and the suffering of the drawing teachers in understanding and using it. In contrast, it showed the advantages of the program, and compared it to the AutoCAD program, and presented a questionnaire by the researcher on a sample of the teachers of the engineering drawing in the degrading schools. program AutoCAD, and therefore, this program does not result in the expected purpose of it, so it became necessary to find an alternative at least to use it as a display of sculptures by engineering drawing teacher, and this alternative is a program Cockle Google sketch up.
\end{abstract}

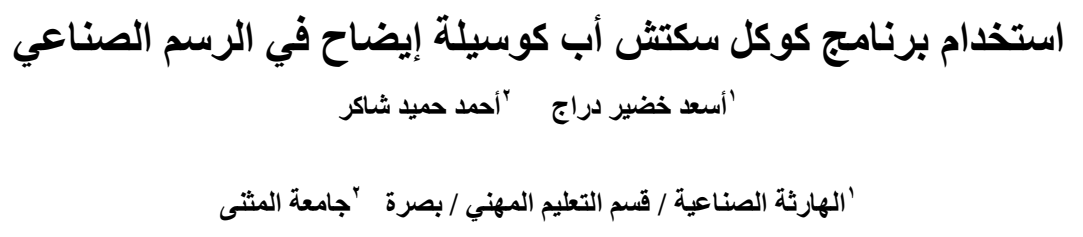

عندما يتعلق الأمر برسم الأشكال ثلاثية الأبعاد فإننا لا نحصل على أسهل من برنامج كوكل سكتش أب Google sketch up, فإن الأهم

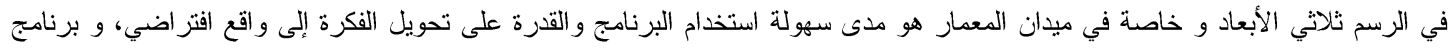
Google sketch up

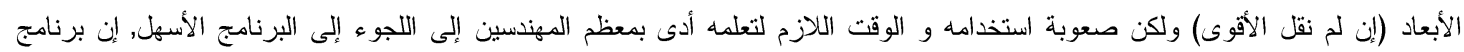

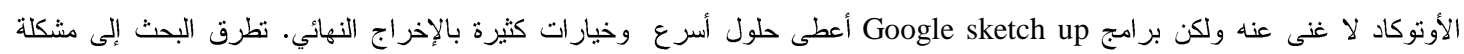

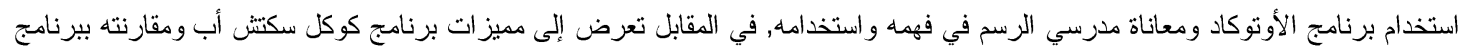

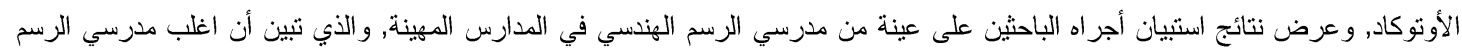

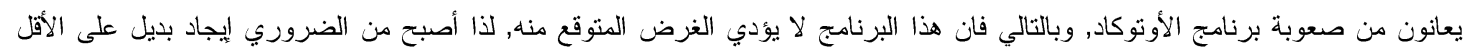

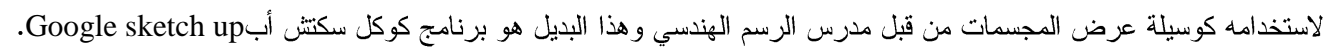

$$
\text { الكلمات الدالة: برنامج كوكل سكتش أب, الرسم الهندسي, الرسم ثلاثي الأبعاد. }
$$


أن أحد أهم ألأهداف الرئيسية لأي برنامج ثلاثي الأبعاد لمدرس الرسم الهندسي والصناعي هو رسم الثكل المنظور وعرضه لطلبته,

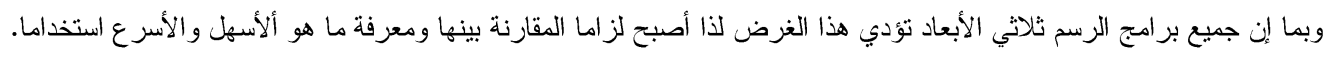
في مدارسنا المهنية يستخدم ددرسوا الرسم الهندي والصناعي برنامج الأوتوكاد لرسم ألأشكال الهندية ولاحظ الباحثين إن اغلب

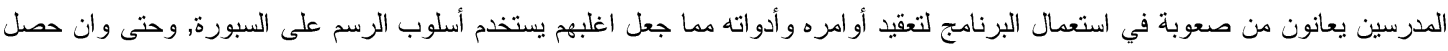

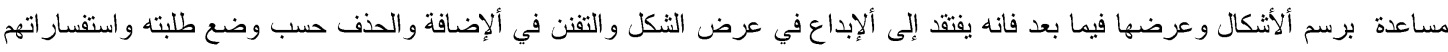

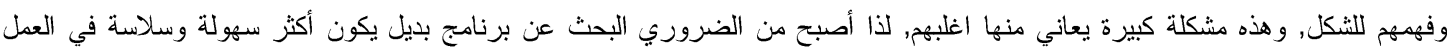

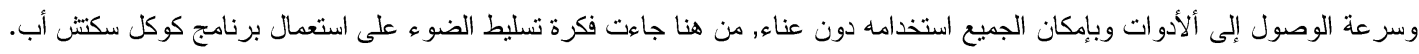

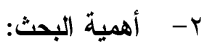

إن للبحث أهمية كبيرة في محاولة تسهيل عمل مدرس الرسم الهندسي في رسم مجسماته ومنظوراته الهندسية بكل سلاسة وسهولة

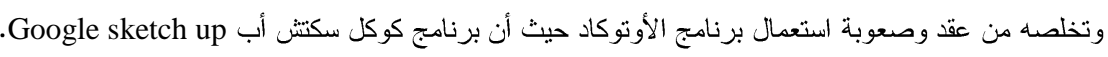
هو برنامج سهل ألانتعمال وأدواته واضحة لا تحتاج إلى تعقيدات ألأو امز والبحث المطول للوصول إلى ألأمر المنشود وبهذا سيسهل كثيرا

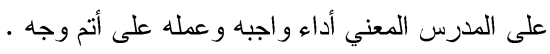

يهدف البحث إلى إلقاء الضوء على برنامج سهل وسريع العمل وهو برنامج كوكل سكتش أب Google sketch up واستعماله كأداة

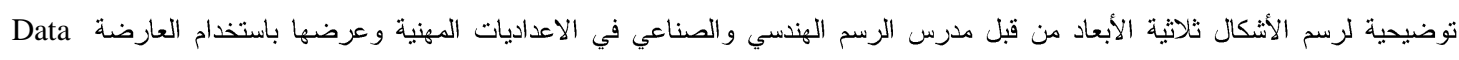

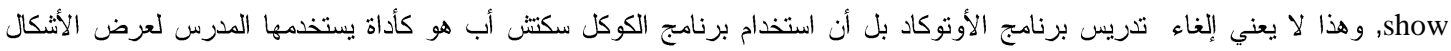

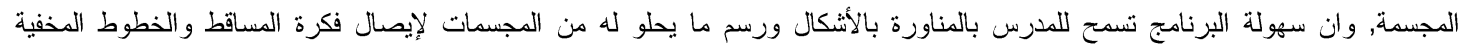

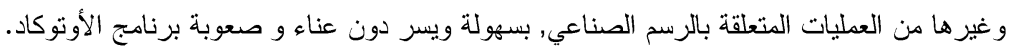

؛ ـ مقدمة:

مما لاثنك فيه إن مادة الرسم الصناعي هو من المواد التي تحتاج إلى استخدام مخيلة واسعة لتخيل وتوقع الثكل بكافة أبعاده وهي بذلك فئل

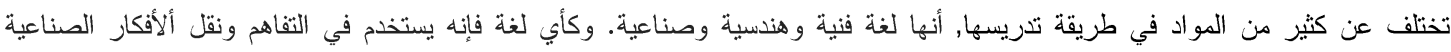
و الهندسية بين المدرس وطلبته, أن استخدام ألأساليب التقليدية في عرض المجسم بو اسطة رسمه على اللوحة (السبورة), يعتبر من ألأساليب القديمة

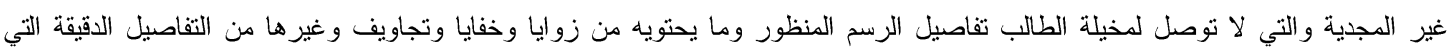

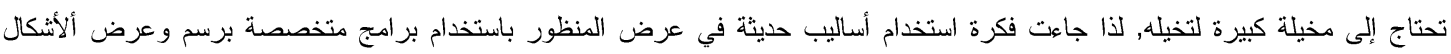

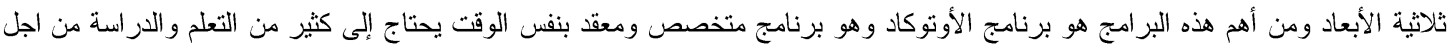

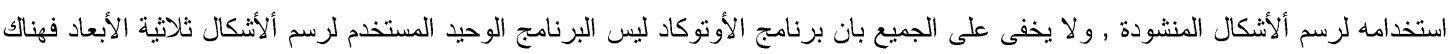

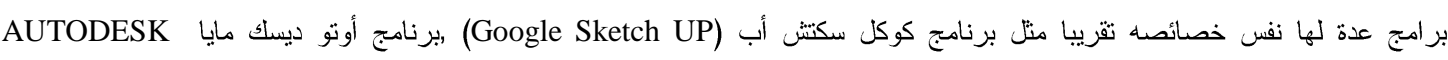
(Maya), و برنامج بلندر (Blender), وكثير من البرامج المتخصصة بالرسم ثلاثي الأبعاد التي تؤدي وظائف مشابهة لبرنامج الأوتوكاد ولكن

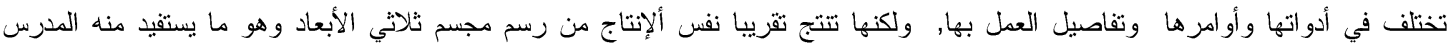

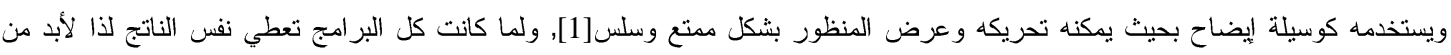
اختيار ما هو أبسطو أسرع للمدرس بحيث يمكنه استخدامه بسهولة ويسر بعيدا عن التعقيدات من هنا انطلقت فكرة البحث, حيث نلاحظ انتشار

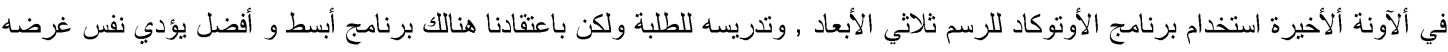

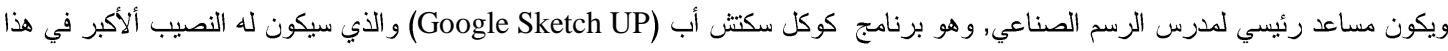

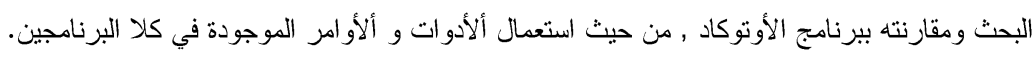

• . برنامج سكتش أب ومميز اته:

وهو من إنتاج شركة كوكل وهو برنامج رسم ثلاثي الأبعاد إن برنامج الرسم الهندسي سكت أب يعتبر من البرامج المتميزة والتي يسهل

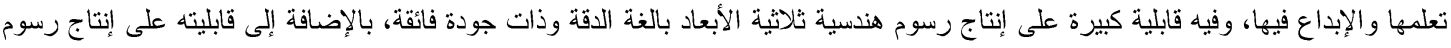
ثنائية الأبعاد بخطوات سهلة وسريعة (ومتخصص أيضا في الرسم المعماري الخارجي والداخلي (الديكور))، من خلال النظر على البرنامج أنه 
ينجز كثيرا في العمل ويمكن ألاعتماد عليه فقط لإخراج عمل معماري كامل بجميع المقاسات والمناظير , البرنامج لا يعد بديلا متكامل لبرامج الرسم ثلاثي الأبعاد ألأخرى وله مميزات ينفرد بها منها: 1- مساحته الصغيرة مقارنة بيرامج الجر افيك الـثزي دي ألأخرى.

إن أغلب برامج الرسم ثلاثي الأبعاد تحتاج إلى مساحة واسعة داخل وحدة الخزن في جهاز الحاسوب فعلى سبيل المثال إن برنامج الأوتوكاد

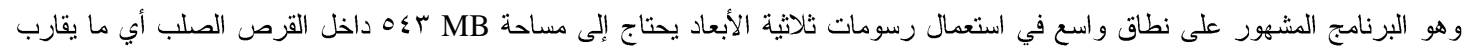

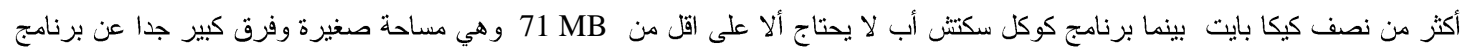
الأوتوكاد[2], بينما يؤدي برنامج كوكل سكتش أب نفس الأداء ونفس النتيجة من حيث الرسم ثلاثي الأبعاد ودقة الرسم من خلال الثكل (1)

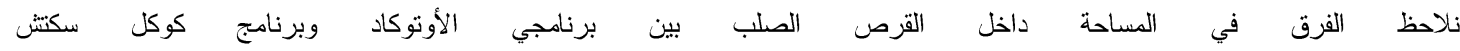

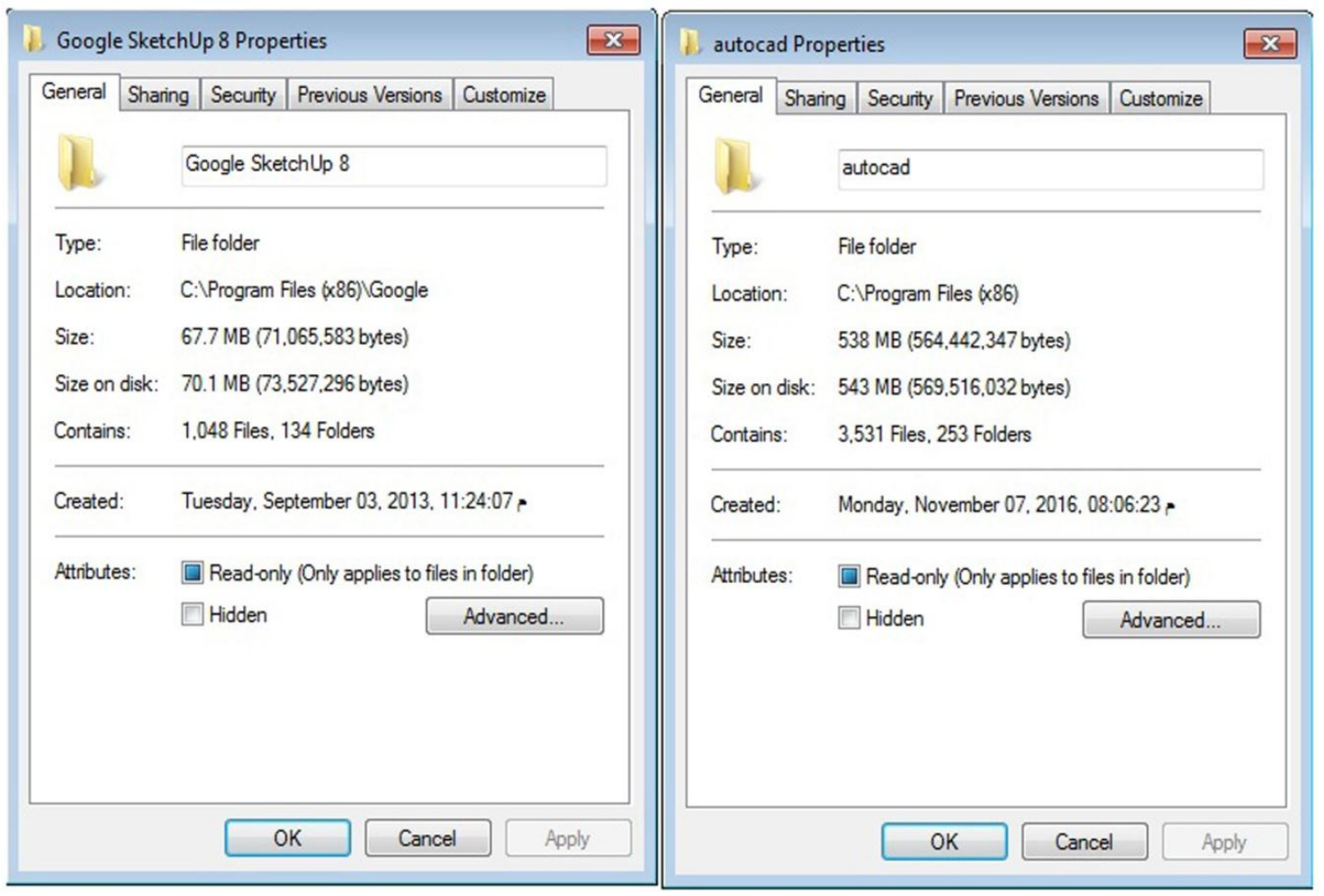

الشكل (1) مساحة برنامجي الأوتوكاد وبرنامج كوكل سكتش أب داخل الحاسوب

ץ- لا يحتاج إمكانيات جهاز ضخمه لتتصيبه والعمل عليه: بما أن برنامج الرسم ثلاثي الأبعاد المستخدم حاليا في مدارسنا المهنية هو برنامج

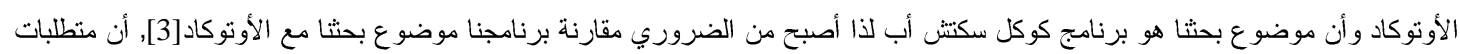

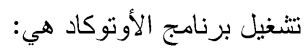

Memory

2 GB (4 GB recommended)

\section{Display resolution}

$1024 \times 768(1600 \times 1050$ or higher recommended $)$ with True Color

Display Card

Supports Windows display adapter capable of $1024 \times 768$ with True Color capabilities

Disk space

Installation $6.0 \mathrm{~GB}$ 


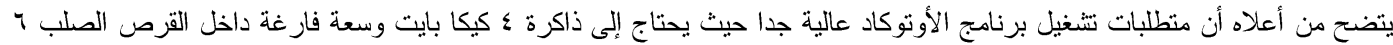

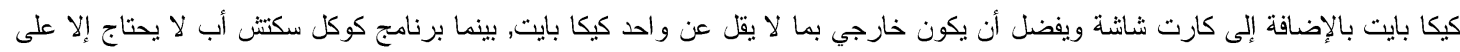

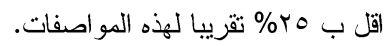
r- سهولته في ألاستخدام فيمكنك تعلمه في خلال أسبوع و احد فقط و البدء في العمل عليه. أن برنامج كوكل سكتش أب هو برنامج سهل وان أدو اته سهل الوصول إليها وبالتالي سلاسة استعمالها , عكس برنامج الأوتوكاد الذي يحتاج إلى دورات مكثيفة ومطولة من اجل استعمال أو امره و أدو اته.

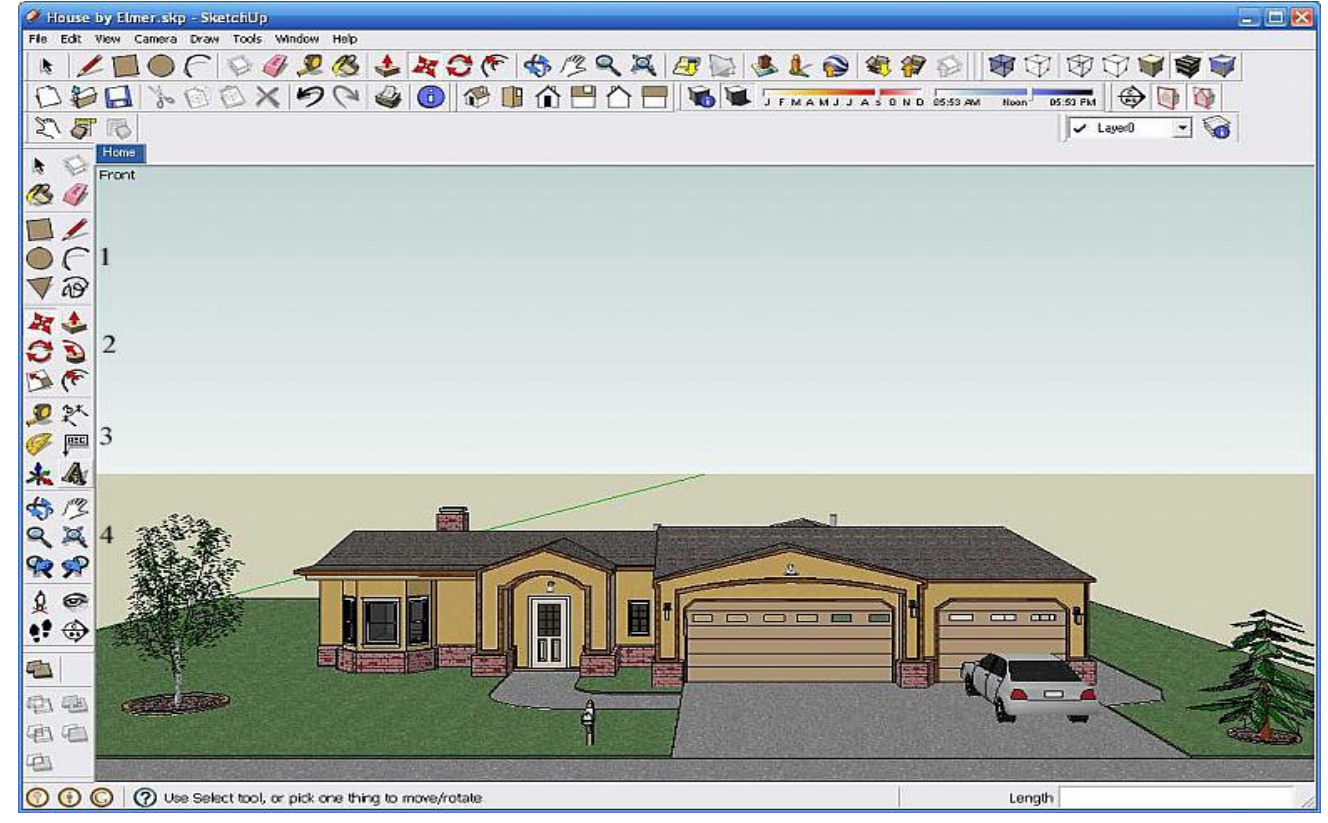

الثكل (ץ) الواجهة الرئيسية لبرنامج كوكل سكتش أب

من خلال الثكل(r) نلاحظ أن الواجهة الرئيسية لبرنامج كوكل سكتش أب هي سهلة وواضحة ولا تحتاج إلى وقت طويل أو لجهد لتعلمها

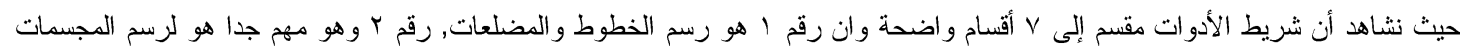

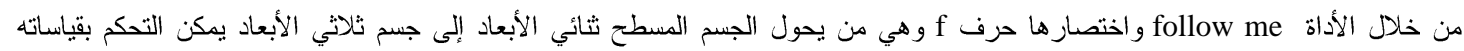

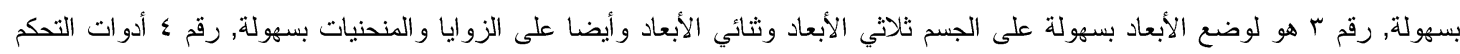

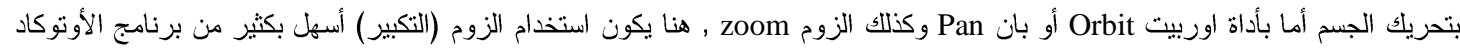

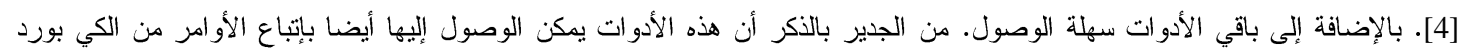
(لوحةالتحكم). 


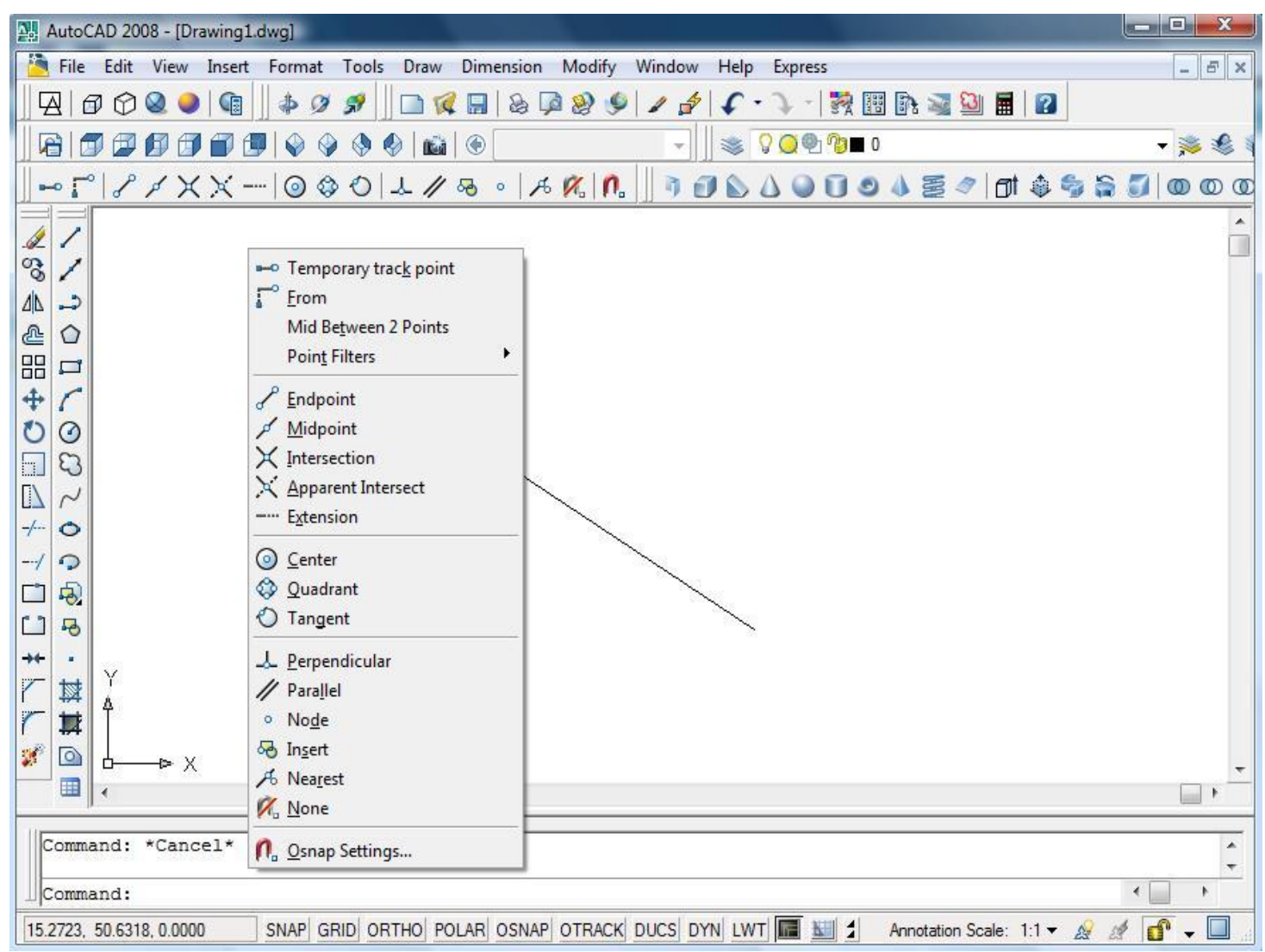

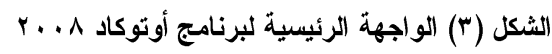

من الثكل (r) نشاهد تعقيدات الأدوات وصعوبة استخدامها ولا يمكن استعمال البرنامج إلامن خلال دورات مختصة بهذا المجال. ع -ألاعتماد عليه كليا في إخـــــــــاج العمل المعماري بالخامات و المقاســـــــات و المساقطو المناظير . 0- إظهار الأبعاد و المقاسات على المجسمات ثلاثية الأبعاد.

تعتبر هذه ميزة جيدة لهذا البرنامج وهو وضع الأبعاد والقياسات بسهولة على المجسم المرسوم لمجرد اختيار أداة القياس و التي هي و اضحة

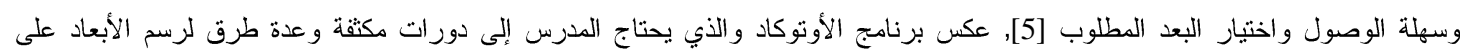
الجسم ثلاثي الأبعاد .

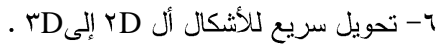

V- متوفر العديد من الموديلات أل Dr الجاهزة خاصة البرنامج لاستخدامها في العمل وتسهيل وتسريع إنجاز العمل. ^- يدعم الكتابة باللغة العربية بدون أي إضافات.

-

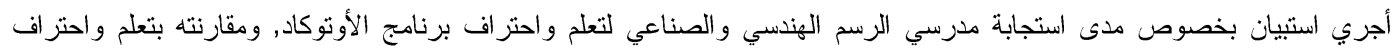

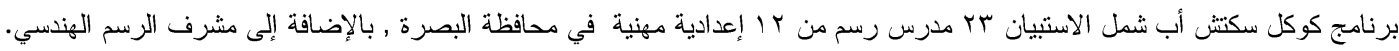

- النتائج - V

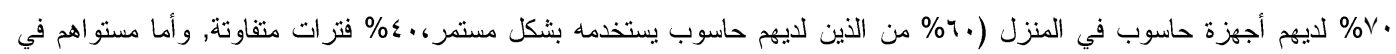

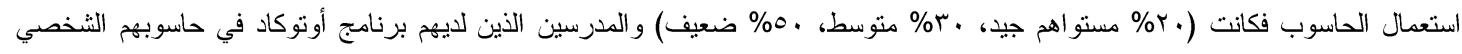

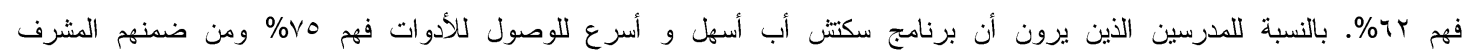

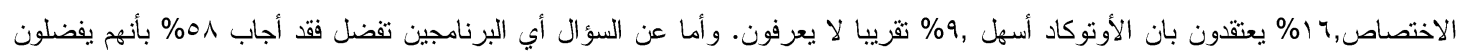




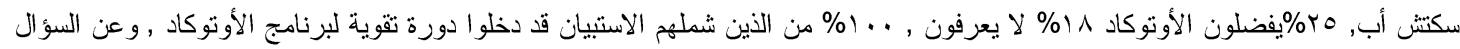

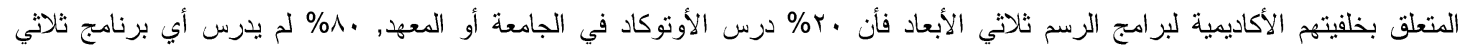

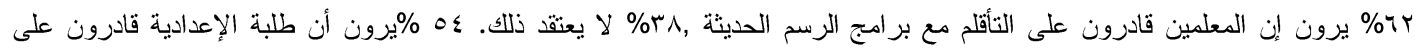

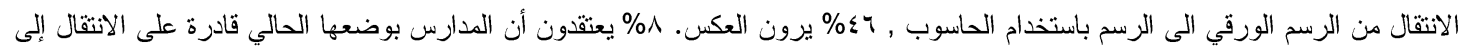

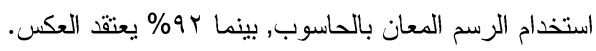

^- الاستنتاجات

يتضح من خلال الاستبيان, أن برنامج الأوتوكاد هو برنامج صعب التعلم وأنه لا يؤدي الغرض المنشود منه في المدارس المهنية , لصعوبة

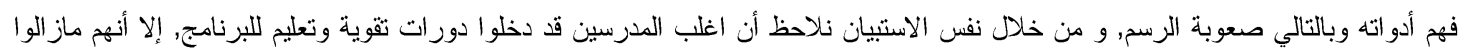

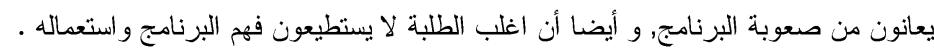

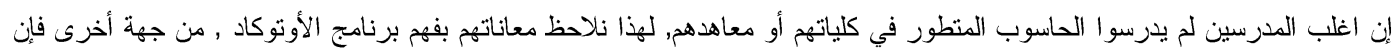
الطلبة يتخرج من الإعدادية وهو لا يسنطيع استعمال برنامج الأوتوكاد لعدة أسباب منها:

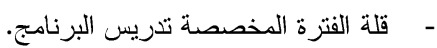

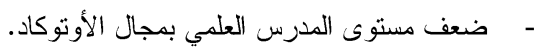

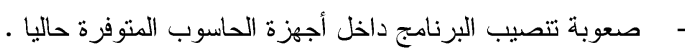

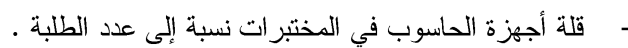

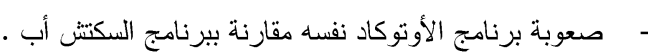
- ضعف مستوى طالب الإعدادية المهنية , بسبب أن الذين يأتون للإعداديات المهنية هم أصحاب المعدلات الضعيفة و منهم من الناجحين في الدور الثالث في المرحلة المتوسطة.

9 - المناقشة

يتضح من نتائج الاستيان ان استخدام برنامج الأوتوكاد من قبل مدرس الرسم يلاقي الكثير من المعوقات يصعب التغلب عليها خاصة وان

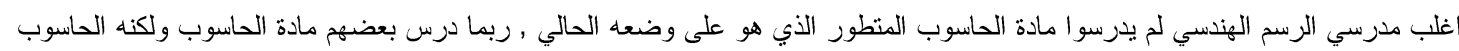

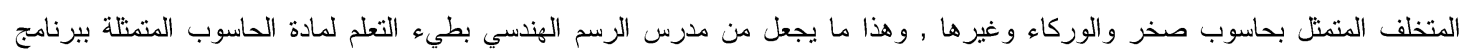

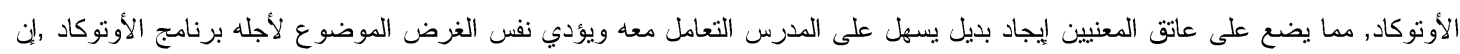

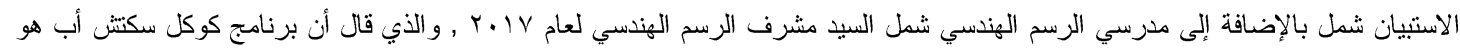

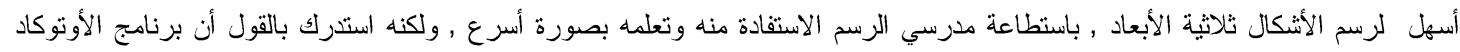

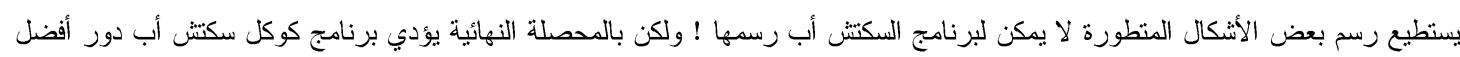
كوسيلة إيضاح للطلبة .

- التوصيات

- استخدام برنامج كوكل سكتش أب من قبل مدرس الرسم الهندسي كوسيلة إيضاح , مع بقاء تدريس برنامج الأوتوكاد في مر احل لاحقة و الالتز ام بالمنهج.

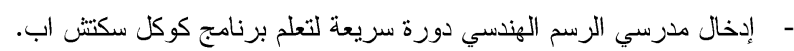

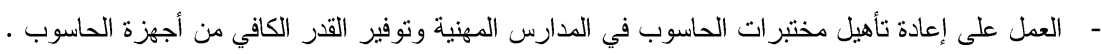

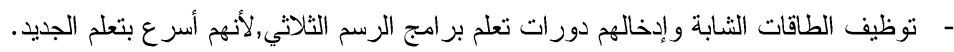
- تخصيص أكثر من حصة مستقلة خاصة بالرسم المعان بالحاسوب.

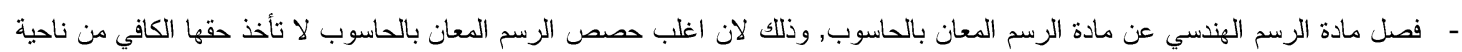
الوقت, حيث يخصص اغلب الوقت للرسم الهندسي و الصناعي. 


\section{CONFLICT OF INTERESTS}

There are no conflicts of interest.

\section{1- References}

[1] J.Lintunen, Streamlining workflow in architectural visualization with Blender and Sketch Up, Thesis, Laurea University of Applied Sciences, Finland, September 2010,14.

[2] Liveri Anna, Xanthacoub Yota, Kailac Maria, The Google Sketch Up Software As A Tool To Promote Creativity In Education In Greece (2002).

[3] Aidan Chopra "Google Sketch Up For Dummies ",10-12. 2007

[4] Anja Kintsch, Google's Sketch Up Software and Individuals with Autism Spectrum Disorders, pp 3. 2009.

[5] Qiang Dong; Yongshou Yang, Research on Sketch Up Application in Graphing of Civil Engineering, July 2014. 